\title{
The Anomalous Thermal Conductivity of Quasi-one-dimensional Hard Disks
}

\author{
Gary P. Morriss \\ School of Physics, University of New South Wales \\ Sydney NSW 2052, Australia \\ E-mail: g.morriss@unsw.edu.au
}

Received: 20 December 2016; revised: 10 March 2017; accepted: 15 March 2017; published online: 15 April 2017

\begin{abstract}
We confirm that the conduction of heat in a system of quasi-one-dimension hard disks, with mechanically connected heat reservoirs of different temperatures, is anomalous. We consider systems of different sizes at the same density with the same externally applied temperature gradient and observe that the anomalous behaviour changes with system size. For systems with less than 1000 disks we find that the heat flux vector varies with the square root of the number of disks whereas for systems with more than 1000 disks the heat flux vector varies with the $2 / 3$ power of the number of disks.
\end{abstract}

Key words: disks, anomalous heat conduction

\section{INTRODUCTION}

The thermal conductivity $\kappa$ is the ratio of the heat flux vector to the negative of the temperature gradient. In a onedimensional system kappa is well known to be anomalous [1] as the conductivity increases with system size $(L)$ or number of particles $(N)$ at constant density. Likewise the dynamics of a one-dimensional system of hard rods is also trivial as each collision results in the interchange of the velocities of the colliding pair. In such a system a velocity input at one boundary exits from the opposite boundary after the full sequence of collisions $i, i+1$ for $i=1, N$ in ascending order. There can be no chaotic dynamics in a one-dimensional system governed by a differential equation. The behavior of the thermal conductivity depends on the details of the system but typically for an anomalous system we observe $\kappa \propto N^{\alpha}$. In fact, it has even been suggested that by changing the coupling of the oscillator chain to the heat reservoirs at the ends, normally thought to be a benign procedure, one can tune the exponent $\alpha$ over a range of values [2]. Another example is the divergence of the thermal conductivity co-efficient with the length, observed in chains of anharmonic oscillators [1, 3, 4] and hard-point gases [5, 6]. The same type of anomaly has been detected for a quasi-1D model consisting of spheres confined in a narrow channel [7], a variant of Sinai's pencase model [9]. The pencase model without heat reservoirs has been proved to be ergodic in four dimensions and hyperbolic in three [10]. The heat reservoirs at the ends were implemented as follows: whenever an extremal particle collided with the reservoir adjoining it, its velocity was randomly drawn from the Boltzmann distribution $P\left(v_{x}, v_{y}\right) \propto v_{x} \exp \left[m\left(v_{x}^{2}+v_{y}^{2}\right) /\left(2 k_{B} T\right)\right]$ where $T$ is the reservoir temperature. Using a narrow channel and a random collision model the conductivity scales as a power law in $N$ over two decades from $N=10$ to $N=1000$, with an exponent very close to the analytical prediction of $1 / 3$, although there is some sensitivity to the mass ratio $m_{1} / m_{2}$. In a following paper [8] the authors find that the scaling depends crucially on the choice of boundary conditions: for periodic boundary conditions as opposed to open boundary conditions with heat baths, the exponent is $1 / 2$.

Posen and Campbell [11] have shown that chaos in the sense of positivity of Lyapunov exponents is neither necessary nor sufficient to guarantee normal transport in 1D lattices. The absence of momentum conservation, even ergodicity of an isolated system is not necessary for normal transport and they demonstrate clearly the validity of the Fourier law in a pseudo-integrable particle chain. Recent ap- 
plications of mode coupling theory [12] predict generically that the thermal conductivity diverges as $N^{1 / 3}$ as the size increases for systems terminated with heat baths at the ends. The $N^{2 / 5}$ dependence is observed in molecular dynamics, which is attributed to a crossover effect. Other mode coupling approaches $[13,15]$ show that the cubic nonlinearity predicts a $t^{-2 / 3}$ decay of the heat current autocorrelation, and thus $\alpha=1 / 3$. It is claimed that this approach with the renormalization group calculation [14] support the idea that the mechanisms yielding anomalous transport in 1D are largely universal. Anomalous transport in one-dimensional translation invariant Hamiltonian systems with short range interactions has been shown [16] to belong in general to the Kardar-Parisi-Zhang (KPZ) universality class [17, 18]. Mode coupling theories developed previously are found to be adequate for weakly nonlinear chains but in need of corrections for strongly anharmonic inter-particle potentials.

In two-dimensions the situation with the thermal conductivity is much less clear however, the dynamics of a twodimensional system of hard disks is chaotic with the resultant loss of information about the initial conditions and an expected decay in correlations. Preliminary results for the system studied here [23] show that the thermal conductivity is anomalous and $\alpha=1 / 2$ but more extensive calculations reported here show that this is not the final story.

\section{THE MODEL}

The model we study here consists of hard sphere particles confined to a long narrow rectangle with periodic boundary conditions in the transverse $(y)$ direction, and heat baths attached at the two ends. For the system to be quasi-onedimensional the width of the rectangle must be less than twice the diameter of the particles, and here both the particle diameter and its mass are equal to one and we use $L_{y}=1.15$, so that a reasonable range of incidence angles at collision is obtained. Here we use a density of 0.8 but this should have no effect on the qualitative results. The transport of energy along the rectangle remains quasi-one-dimensional, with the transverse degree of freedom serving as an additional velocity randomizing effect.

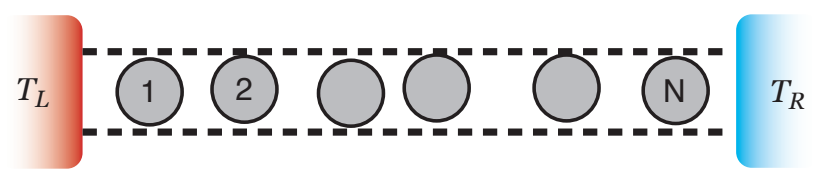

Fig. 1. The quasi-one-dimensional (QOD) hard-disk system. The width of the system $L_{y}=1.15<2$ so that the disks remain in the same order. The system is periodic in the $y$ direction so has images above and below

In 2007 a deterministic thermal reservoir was introduced that coupled the QOD system (see fig.(1)) of hard disks to temperature reservoirs dynamically by changing the collision rule at the reservoir boundary [19]. For a collision with a reservoir boundary the tangential $y$-component of momentum is unchanged but the normal $x$-component after collision becomes

$$
m v_{x}^{\prime}=\epsilon m v_{r e s}-(1-\epsilon) m v_{x},
$$

where $m v_{r e s}$ is a reservoir momentum related to the reservoir temperature by $m v_{\text {res }}=\sqrt{2 T_{\text {res }}}$ and $\epsilon$ is a reservoir coupling parameter which we typically take to be equal to 0.5 . The reservoir momentum is always directed into the system so $T_{L}$ is positive and $T_{R}$ is negative. As $\epsilon \rightarrow 0$ the system decouples from the reservoir and the boundary becomes a hard wall, and as $\epsilon \rightarrow 1$ the incoming momentum is replaced by the reservoir momentum. For systems with energy input into the $x$-direction only, the energy in $x$ and $y$-directions can interchange locally through the collisional dynamics achieving some measure of local thermodynamic equilibrium at each particle. The quasi-one-dimensional (QOD) system that we use here can be considered to be intermediate between 1 and 2 dimensions as the width $L_{y}<2$ maintaining the fixed particle ordering of a 1-dimensional system. As the size of the second dimension of the container holding the hard disks increases from one hard disk diameter to two hard disk diameters we go from one-dimensional hard rods with fixed particle ordering to a full two-dimensional system at more than two diameters where particle ordering disappears. As such we can imagine the QOD system as a transition between one and two-dimensional behaviour which we can in principle explore by increasing the value of $L_{y}$.

A recent study of this system in contact with two reservoirs of the same temperature [22, 24, 27] has shown that the active mechanical coupling leads to entropy production near each reservoir which then flows into the reservoir. These effects are local and involve a limited number of boundary layer particles regardless of the system size.

Treating this as a dynamical system the Lyapunov spectrum and Lyapunov vectors, both Gram-Schmidt and covariant, have been studied extensively [26] and field theory derivations of the structure of the Lyapunov modes has been obtained [25].

It is a very clean and essentially exact calculation for a computer. The interaction with the heat reservoirs is mechanical and has no random elements. There is no thermostatting apart from that implied by the interaction of boundary particles with the reservoirs. The statistical properties of the system can be obtained by calculating the velocity distributions of each particle and various particle-particle correlation functions. In this way the densities and fluxes of energy and entropy can be calculated with almost no approximations.

\section{1. Microscopic Heat flux vector}

For a system of spherical particles the microscopic representation for the instantaneous heat flux vector at position $\mathbf{r}$ 
and at time $t$ is given by $[20,21]$

$$
\begin{aligned}
\mathbf{J}_{Q}(\mathbf{r}, t) & =\sum_{i=1}^{N} U_{i} \mathbf{v}_{i} \delta\left(\mathbf{r}-\mathbf{r}_{i}\right) \\
& -\frac{1}{2} \sum_{i, j}^{N} \mathbf{r}_{i j} \mathbf{F}_{i j} \cdot\left(\mathbf{v}_{i}+\mathbf{u}\left(\mathbf{r}_{i}\right)-\mathbf{u}(\mathbf{r})\right) \\
& \times \int_{0}^{1} d \lambda \delta\left(\mathbf{r}-\mathbf{r}_{i}-\lambda \mathbf{r}_{i j}\right)
\end{aligned}
$$

where $U_{i}=\frac{1}{2} m\left(\mathbf{v}_{i}-\mathbf{u}(\mathbf{r})\right)^{2}$ is the internal energy of particle $i, \mathbf{u}(\mathbf{r})$ is the local streaming velocity at position $\mathbf{r}$ and $\mathbf{u}\left(\mathbf{r}_{i}\right)$ is the local streaming velocity at the position of particle $i$. For this QOD system the local streaming velocity is zero everywhere. We define the vectors $\mathbf{r}_{i j}=\mathbf{r}_{j}-\mathbf{r}_{i}$ and $m \mathbf{v}_{i j}=m \mathbf{v}_{j}-m \mathbf{v}_{i}$. For hard core particles the interaction force is an impulse $\mathbf{F}_{i j}=\left(\hat{\mathbf{r}}_{i j} \cdot \mathbf{v}_{i j}\right) \hat{\mathbf{r}}_{i j} \delta\left(t-t_{i j}\right)$, where $t_{i j}$ is the time at which a collision occurs between particles $i$ and $j$, and $\hat{\mathbf{r}}_{i j}$ is the unit vector in the direction of $\mathbf{r}_{i j}$.

In the integral in Eq. (II. 1.), the delta function moves along the line joining the centre of particle $i, \mathbf{r}_{i}$ with the centre of particle $j, \mathbf{r}_{j}$, as $\lambda$ goes from 0 to 1 , so to approximate this integral we use a single strip, thus half the contribution at $\mathbf{r}_{i}$ and half at $\mathbf{r}_{j}$. This is analogous to assigning half the potential energy of interaction to each particle for particles interacting with a continuous potential. The one strip approximation to the integral is $\frac{1}{2}\left(\delta\left(\mathbf{r}-\mathbf{r}_{i}\right)+\delta\left(\mathbf{r}-\mathbf{r}_{j}\right)\right)$ so Eq. (II. 1.) becomes

$$
\begin{aligned}
\mathbf{J}_{Q}(\mathbf{r}, t) & =\sum_{i=1}^{N} U_{i} \mathbf{v}_{i} \delta\left(\mathbf{r}-\mathbf{r}_{i}\right) \\
& -\frac{1}{4} \sum_{i, j}^{N} \hat{\mathbf{r}}_{i j}\left(\hat{\mathbf{r}}_{i j} \cdot \mathbf{v}_{i j}\right) \hat{\mathbf{r}}_{i j} \cdot\left(\mathbf{v}_{i}+\mathbf{v}_{j}\right) \\
& \times \delta\left(t-t_{i j}\right)\left(\delta\left(\mathbf{r}-\mathbf{r}_{i}\right)+\delta\left(\mathbf{r}-\mathbf{r}_{j}\right)\right)
\end{aligned}
$$

In this form it is clear how the individual contributions are assigned to each particle. The kinetic contribution is at $\mathbf{r}_{i}$ while there are two potential contributions, one at $\mathbf{r}_{i}$ and the other at $\mathbf{r}_{j}$. Notice that if $\mathbf{v}_{i}+\mathbf{v}_{j}=0$ there is no collisional energy transfer so the transfer of energy requires particles $i$ and $j$ to have velocities of different magnitude.

We will be interested in both the total heat flux $\mathbf{J}_{Q}(t)$ and the heat flux vector at the position of each particle $\mathbf{J}_{Q}\left(\mathbf{r}_{i}, t\right)$. The heat flux at the position of each particle will determine whether energy continuity is satisfied everywhere in the system as, on average, the same amount of energy must passes through any vertical line regardless of its position. For the QOD system the local heat flux $\mathbf{J}_{Q}\left(\mathbf{r}_{i}, t\right)$ has potential contributions from two sources, either from a collision of particles $i$ and $i+1$ or from a collision of particles $i-1$ and $i$.

Returning to the heat current density in Eq. (II. 1.), we can define the heat current at some arbitrary $x$ value. Instantaneously, there is only a kinetic contribution if a particle has its coordinate $x_{i}=x$, and there is only a potential contribution if two particles collide where for one $x_{i}<x$ and for the other $x_{j}>x$ so that the line of delta functions in Eq. (II. 1.) has one at position $x$. The time average of this instantaneous quantity must satisfy the continuity equation.

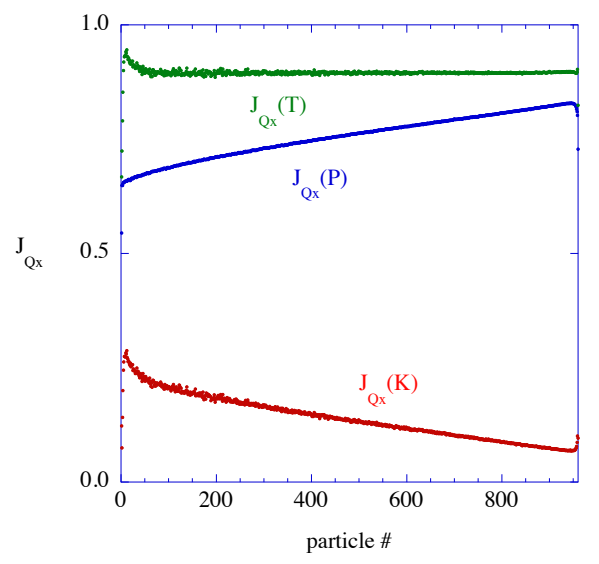

Fig. 2. Heat flux continuity across the QOD hard-disk system. The red points are the kinetic contribution, the blue points are the potential contribution and the green points are the total heat flux vector at the position of each particle. These results are for a system of 960 disks but similar results are observed at other system sizes

To produce a nonequilibrium steady state it is sufficient to have reservoirs of different temperature on each side of the QOD system. The energy entering the system from a boundary with reservoir momentum $m v_{\text {res }}$ during a collision with a particle of incoming momentum $m v_{x}$ is given by

$$
\begin{aligned}
& \Delta e_{\text {res }}=\frac{m}{2}\left({v^{\prime}}_{x}^{2}-v_{x}^{2}\right)= \\
= & \frac{m \epsilon}{2}\left[\epsilon v_{r e s}^{2}+2(\epsilon-1) v_{r e s} v_{x}+(\epsilon-2) v_{x}^{2}\right] .
\end{aligned}
$$

The time average of this quantity gives the flux of energy through the system, so for a steady state the energy flux from the left-hand side reservoir into the system $\Delta e_{L}$ must be equal in magnitude but opposite in sign to the heat flux from the system into the right-hand reservoir $\Delta e_{R}$. Therefore in a nonequilibrium steady state the following time averages must be equal

$$
\left\langle\Delta e_{L}\right\rangle=\left\langle J_{Q x}\right\rangle L_{y}=-\left\langle\Delta e_{R}\right\rangle .
$$

where $J_{Q x}=\mathbf{J}_{Q}(x, t)$. The energy flux at the boundaries is controlled by both $v_{\text {res }}$ (or the reservoir temperature) and the value of $\epsilon$, going to zero as $\epsilon \rightarrow 0$ and the reservoirs become disconnected from the system.

\section{THE RESULTS AND CONCLUSIONS}

We fix the right-hand side reservoir temperature to be $T_{R}=2$ and the externally applied temperature gradient 
Tab. 1. The simulation state points considered. For all systems the density $\rho=0.8$ and $T_{R}=2$. As $T_{R}=2$ is fixed and $N$ varies, the left-hand side temperature $T_{L}$ varies so that the temperature gradient $\nabla T=-0.01150$ remains fixed. $B$ is the number of blocks of $10^{9}$ collisions used at each particular state point

\begin{tabular}{c|cc|ccc|c}
\hline$N$ & $T_{L}$ & $L_{x}$ & $\Delta e_{L}$ & $J_{Q} \times L_{y}$ & $\Delta e_{R}$ & $B$ \\
\hline 80 & 2 & 86.957 & $0.2868 \pm 0.0002$ & $0.2835 \pm 0.0002$ & $0.2868 \pm 0.0002$ & 8 \\
160 & 4 & 173.91 & $0.3959 \pm 0.0005$ & $0.3959 \pm 0.0005$ & $0.3936 \pm 0.0005$ & 8 \\
320 & 6 & 347.83 & $0.557 \pm 0.0010$ & $0.557 \pm 0.0010$ & $0.555 \pm 0.0010$ & 7 \\
640 & 10 & 695.65 & $0.8098 \pm 0.0013$ & $0.8086 \pm 0.0013$ & $0.8098 \pm 0.0012$ & 18 \\
960 & 14 & 1043.5 & $1.0304 \pm 0.0032$ & $1.0303 \pm 0.0028$ & $1.0303 \pm 0.0027$ & 20 \\
1280 & 18 & 1391.3 & $1.2325 \pm 0.0044$ & $1.2318 \pm 0.0043$ & $1.2330 \pm 0.0043$ & 16 \\
1920 & 26 & 2087.0 & $1.6001 \pm 0.0068$ & $1.6004 \pm 0.0067$ & $1.607 \pm 0.011$ & 16 \\
2560 & 34 & 2782.6 & $1.936 \pm 0.012$ & $1.936 \pm 0.008$ & $1.936 \pm 0.007$ & 18 \\
3840 & 50 & 4173.9 & $2.543 \pm 0.050$ & $2.563 \pm 0.033$ & $2.578 \pm 0.036$ & 14 \\
5120 & 66 & 5565.2 & $3.127 \pm 0.056$ & $3.13 \pm 0.03$ & $3.130 \pm 0.016$ & 24 \\
10240 & 130 & 11130 & $5.13 \pm 0.36$ & $5.03 \pm 0.23$ & $4.952 \pm 0.032$ & 24 \\
\hline
\end{tabular}

$\nabla T=\left(T_{R}-T_{L}\right) / L_{x}$ to be -0.01150 , so that the value of $L_{x}$ (or the system size) determines left-hand side reservoir temperature $T_{L}$. We consider the heat flux vector at the position of each particle and its two contributions, the kinetic and potential components in Fig.(2). The kinetic heat flux vector varies with particle position, as does the potential heat flux vector, but the two contributions combine to give a constant value, except in a region near the hot reservoir. This anomaly appears to come from the kinetic contribution to the heat flux and suggests that boundary effects near the hot reservoir extend further into the system than at the cold reservoir.

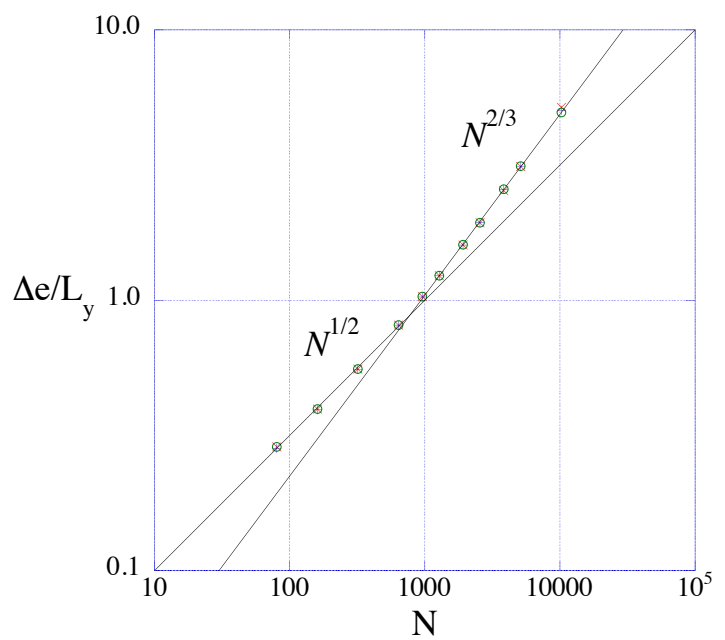

Fig. 3. The $x$ component of the heat flux vector times $L_{y}, J_{Q} L_{y}$ (blue plus signs), the energy flux into the system from the left-hand reservoir $\Delta e_{L}$ (red crosses) and the energy flux out of the system into the right-hand reservoir $-\Delta e_{R}$ (green circles). For all systems the three different methods give the same results. Very close to $N=1000$ there is a change in the anomalous behaviour of the energy flux from $N^{1 / 2}$ to $N^{2 / 3}$
An overview of simulation state points is given in Table. (1). The accuracy of the results is estimated by calculating the standard deviation from $N$ blocks and extrapolating to $N=1$. Thus if $\sigma(N)$ is the standard deviation calculated from $N$ block averages then the standard deviation of the full data set is estimated as $\sigma(1)=\lim _{N \rightarrow \infty} \sigma(N) / \sqrt{N}$.

The results in Fig. (3) here show that a QOD system of hard disks with deterministic thermostat interaction has a different anomalous behaviour to a similar system [7] with random collisions and reservoir momenta chosen randomly from a canonical distribution at constant temperature. Further this QOD model, with exact collision dynamics within the system, shows different anomalous behaviour depending upon system size. At $N=1000$ there appears to be the transition point between $N^{1 / 2}$ and $N^{2 / 3}$ behaviours. Mode coupling theories seem to agree with the results observed in simulations when the correct couplings are input. Here it seems that the coupling terms change with system size so perhaps the representation as fluid elements rather than as individual atoms occurs at 1000 particles.

\section{References}

[1] S. Lepri, R. Livi, A. Politi, Thermal conduction in classical low-dimensional lattices, Phys. Rep. 377, 1 (2003).

[2] A. Dhar, Heat conduction in the disordered harmonic chain revisited, Phys. Rev. Lett. 86, 5882 (2001).

[3] S. Lepri, R. Livi, A. Politi, Heat conduction in chains of nonlinear oscillators, Phys. Rev. Lett. 78, 1896 (1997).

[4] S. Lepri, R. Livi, A. Politi, On the anomalous thermal conductivity of one-dimensional lattices, Europhys. Lett. 43, 271 (1998).

[5] T. Hatano, Heat conduction in the diatomic Toda lattice revisited, Phys. Rev. E 59, R1 (1999).

[6] P. Grassberger, W. Nadler, L. Yang, Heat conduction and entropy production in a one-dimensional hard-particle gas, Phys. Rev. Lett. 89, 180601 (2002). 
[7] J.M. Deutsch, O. Narayan, One-dimensional heat conductivity exponent from a random collision model Phys. Rev. E 68, 010201 (2003).

[8] J.M. Deutsch, O. Narayan, Correlations and scaling in onedimensional heat conduction Phys. Rev. E 68, 041203 (2003).

[9] Ya.G. Sinai, N.I. Chernov, Ergodic properties of certain systems of two-dimensional disks and 3-dimensional balls, Russ. Math. Surveys 42, 181 (1987).

[10] N. Simányi, D. Szász, The k-property of hamiltonian systems with restricted hard ball interactions Math. Res. Lett. 2, 751 (1995).

[11] T. Prosen, D.K. Campbell, Normal and anomalous heat transport in one-dimensional classical lattices, CHAOS 15, 015117 (2005).

[12] J-S Wang, B. Li, Mode-coupling theory and molecular dynamics simulation for heat conduction in a chain with transverse motions, Phys. Rev. E 70, 021204 (2004).

[13] L. Delfini, S. Lepri, R. Livi, A. Politi, Self-consistent modecoupling approach to one-dimensional heat transport, Phys. Rev. E 73, 060201(R) (2006).

[14] O. Narayan, S. Ramaswamy, Anomalous heat conduction in one-dimensional momentum-conserving systems, Phys. Rev. Lett. 89, 200601 (2002).

[15] L. Delfini, S. Lepri, R. Livi and A. Politi, Anomalous kinetics and transport from $1 D$ self-consistent mode-coupling theory, J. Stat. Mech. 7, P02007 (2007).

[16] H. van Beijeren, Exact Results for Anomalous Transport in One-Dimensional Hamiltonian Systems, Phys. Rev. Lett. 108 180601 (2012).
[17] M. Prähofer, H. Spohn, Exact scaling functions for onedimensional stationary KPZ growth, J. Stat. Phys. 115, 255 (2004).

[18] T. Sasamoto, H. Spohn, Superdiffusivity of the 1D Lattice Kardar-Parisi-Zhang Equation, J. Stat. Phys. 137, 917 (2009).

[19] T. Taniguchi, G.P. Morriss, Lyapunov Modes for a Nonequilibrium System with a Heat Flux, Comptes Rendus Physique, 8, 625 (2007).

[20] J.A. McLennan, Introduction to Non-equilibrium Statistical Mechanics, Prentice-Hall, Englewood Cliffs NJ, 1989.

[21] D.J. Evans, G.P. Morriss, Statistical Mechanics of Nonequilibrium Liquids, 2nd Edition, Cambridge University Press, Cambridge, 2008.

[22] G.P. Morriss, D. Truant, Deterministic Thermal Reservoirs, Entropy, 14, 1011 (2012).

[23] G.P. Morriss, D. Truant, Dissipation and entropy production in deterministic heat conduction of quasi-one-dimensional systems, Phys. Rev. E, 87, 062144 (2013).

[24] G.P. Morriss, D.P. Truant, A Review of the Hydrodynamic Lyapunov Modes of Hard Disk Systems, J. Phys. A. 46254010 (2013).

[25] T. Chung, D. Truant, G.P. Morriss, Lyapunov Modes as Fields, Phys. Rev. E 83, 046216 (2011).

[26] D.P. Truant, G.P. Morriss, Backward and covariant Lyapunov vectors and exponents for hard disk systems with a steady heat current, Phys. Rev. E 90, 052907 (2014).

[27] G.P. Morriss, Calculating the Local Nonequilibrium Configurational Entropy in Quasi-one-dimensional Heat Conduction, Molecular Simulations 42(6-7) 474-483(2016).

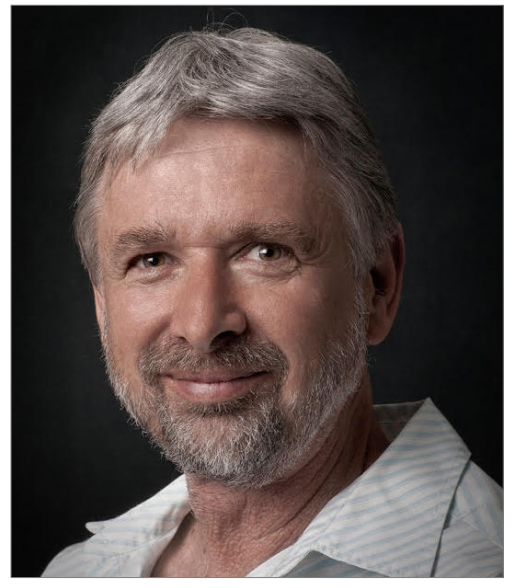

Gary Morriss received his $\mathrm{PhD}$ in applied mathematics from University of Melbourne 1981. After post-doctoral appointments at Cornell University and the Australian National University he gained a permanent position at the School of Physics, UNSW. In 2016 he retired and is currently Professor emeritus at UNSW. His research interests are in nonequilibrium statistical mechanics and dynamical systems. He has held visiting positions at IPST, Maryland; CECAM, l'ENS de Lyon; EPFL, Switzerland; Technical University, Berlin; and CEA-Saclay, France. 